\title{
Extracting information from asset prices: The methodology of EMU calculators
}

\author{
Carlo A. Favero ${ }^{\mathrm{a}, \mathrm{b}, *}$, Francesco Giavazzi ${ }^{\mathrm{a}, \mathrm{b}}$, Fabrizio Iacone $^{\mathrm{a}}$, \\ Guido Tabellini ${ }^{\mathrm{a}, \mathrm{b}}$
}

${ }^{a}$ IGIER, Università Bocconi, Via Salasco 5, 20136 Milan, Italy

${ }^{\mathrm{b}}$ Centre for Economic Policy Research, London, UK

Received 1 July 1997; accepted 1 March 1999

\begin{abstract}
This paper develops a particular technique for extracting market expectations from asset prices. We use the term structure of interest rates to estimate the probability the market attaches to the event that a country, Italy, joins the European Monetary Union at a given date. The case of Italy is interesting because in the survey regularly conducted by Reuters, the probability that Italy joins EMU in 1999 has fluctuated, in the first months of 1997 , between 0.07 and 0.15 , while, during the same period, the measures computed by financial houses - which are based on the term structure of interest rates - ranged between 0.5 and 0.8 . The paper proposes a new method for computing these probabilities, and shows that the discrepancies between survey and market-based measures are not the result of market inefficiencies, but depend on an incorrect use of the term structure to compute probabilities. The technique proposed in the paper can also be used to distinguish between convergence of probabilities and convergence of fundamentals, that is to find out whether an observed reduction in interest rate spreads signals a higher probability of joining EMU at a given date, or simply reflects improved fundamentals. It could also be applied, more generally, to extract from assets prices, information on imminent changes in an exchange rate regime. (C) 2000 Elsevier Science B.V. All rights reserved.
\end{abstract}

JEL classification: E43; E52

Keywords: Term structure of interest rates; Expectational model; Probabilities of entering EMU

\footnotetext{
* Corresponding author. IGIER, Università Bocconi, Via Salasco 5, 20136 Milan, Italy. Tel.: +39-02-58363306; fax: +31-02-58363302.

E-mail address: carlo.favero@uni-bocconi.it (C.A. Favero).
} 


\section{Introduction}

Extracting market expectations from asset prices is a question which has recently attracted a great deal of interest (for recent reviews see Söderlind and Svensson, 1997; Bates, 1998). This paper looks at a specific example: how can the term structure be used to estimate the probability the market attaches to the event that a country, Italy, joins the European Monetary Union at a given date. We have been drawn towards this example observing the striking difference that existed between the surveys regularly conducted among market participants, and the probabilities estimated extracting information from the term structure. Table 1 reports six observations (over the interval January to October 1997) on the probability that Italy joins the EMU on January 4, 1999. Up to the summer, the surveys conducted by Reuters show a remarkably stable assessment of Italy's chances - ranging from a minimum of 0.07 in February, to a maximum of 0.17 the previous month. ${ }^{1}$ In the same table we report the probabilities computed accordingly to the J.P. Morgan EMU Calculator, regularly published in the Financial Times, and the Credito Italiano EMU Calculator, published in the Italian daily Corriere della Sera. The probabilities computed using these two techniques are very similar, but quite distant from the results of the survey. In particular, the survey reached a minimum in February, which does not coincide with the month in which the 'calculators' show a minimum; both the survey and the 'calculators' show a maximum in January, but the probability computed using the 'calculator' is four times larger than that of the survey. After the summer, the acceleration of the convergence process reduces the discrepancies between the three indicators.

Table 1

Probability that Italy joins the EMU from the start, on January 4, 1999

\begin{tabular}{lllllll}
\hline & 15 Jan 97 & 15 Feb 97 & 15 Mar 97 & 15 Apr 97 & 15 May 97 & 15 Oct 97 \\
\hline $\begin{array}{l}\text { Reuters EMU } \\
\text { survey }\end{array}$ & 0.17 & 0.07 & 0.12 & 0.15 & 0.14 & 0.74 \\
$\begin{array}{l}\text { Credito Ital. } \\
\text { EMU calculator }\end{array}$ & 0.78 & 0.70 & 0.54 & 0.55 & 0.58 & 0.90 \\
$\begin{array}{l}\text { J.P. Morgan } \\
\text { EMU calculator }\end{array}$ & 0.70 & 0.61 & 0.51 & 0.56 & 0.58 & 0.90 \\
\hline
\end{tabular}

\footnotetext{
${ }^{1}$ Reuters polls each month 43 experts at banks, research houses, think tanks, universities and employers' associations across Europe. The list of panelists is available from the Reuters code $\langle$ EMUPOLL37〉. Poll details are on Reuters pages $\langle$ EMUPOLL30〉 to $\langle$ EMUPOLL37〉.
} 
The aim of this paper is to investigate the sources of the discrepancies observed up to the summer of 1997 . They could be related to the way in which these 'calculators' are constructed; alternatively they could be the result of market inefficiencies, or of risk premia terms. In the second and third sections of the paper we discuss the construction of a 'calculator', spelling out the assumptions that are needed in order to produce an estimate of probabilities. The technique we develop also provides a simple way to investigate a different, although related, question: whether the convergence between Italian and German interest rates, observed since the third-quarter of 1996, was the result of converging fundamentals, or of a change in the assessment of Italy's chances to join EMU, related to market sentiment but not to fundamentals. Finally, in the last section of the paper, we appraise the 'EMU Calculators'.

These issues will remain relevant even after the start of the EMU, with reference to late entrants. The technique we propose could also be applied more generally, to extract information on imminent changes in an exchange rate regime from asset prices. $^{3}$

\section{The construction of an EMU calculator}

The premise of this exercise is that the German yield curve is to be taken as the benchmark for the euro curve after January 4, 1999. Hence, we start by estimating the term structure of spot rates for Italy and Germany. From it we then extract the term structure of instantaneous forward rates for each country. ${ }^{4}$ We interpret this forward curve as the sequence of overnight rates expected to prevail at any date in the future. Forward rates are interest rates on investments made at a future date, the settlement date, and expiring at a date further into the future, the maturity date. Instantaneous forward interest rates are the limit as the maturity date and the settlement date approach one another. The relationship between a 'yield-to-maturity' and the 'instantaneous forward rate' at that maturity is thus analogous to the relationship between marginal and average cost. The curve of instantaneous forward rates thus lies above the curve of spot rates,

\footnotetext{
${ }^{2}$ Since writing the first version of this paper, we became aware of three other papers on this same issue. Lund (1998), whose methodology is similar to our own; Butler and Cooper (1997), who use instead the information contained in exchange rate options; Bates (1998) who provides an evaluation of different approaches including the prices of Arrow-Debreu securities whose payoff is contingent upon the country being admitted into the EMU.

${ }^{3}$ Although the problem we study is similar to that investigated by Flood and Marion (1983), and Flood and Garber (1983) - namely how asset prices incorporate the expectation of a regime change - those papers do not exploit the information contained in the term structure.

${ }^{4}$ The idea of using forward rates to compute EMU calculators has been originally suggested by De Grauwe (1996) and further developed by Weidman (1996).
} 
when this is positively sloped, and below the curve of spot rates, when this is negatively sloped. ${ }^{5}$

If the pure expectations model is valid, and there is no term premium, then instantaneous forward rates at future dates can be interpreted as the overnight spot rates expected to prevail at those future dates. If we think of the overnight rate as the rate controlled by the central bank, then the curve of instantaneous forward rates can be interpreted as an indicator of expected future monetary policy. Instantaneous forward rates are of particular interest in judging the likelihood of a country joining the EMU, since in the monetary union the overnight rate will be the same for all participating countries.

At any future date $T$ after December 31, 1998, one of the following two complementary events must be true: either at date $T$ Italy belongs to the EMU, or else at date $T$ Italy does not belong to the EMU - we treat Italy being excluded from the EMU, or the fact that there is no EMU at all, as the same event here. In the first event, the date $T$ Italian instantaneous forward rate coincides with that of Germany. The future German forward rate is observed and can be extracted from the German yield curve. In the second event, the Italian instantaneous forward rate will be determined by Italian monetary policy: it will reflect Italian and international fundamentals, and it will not necessarily coincide with the German instantaneous forward rate. Estimating this hypothetical Italian instantaneous forward rate for date $T$, if at $T$ Italy is out of the EMU, is the main problem we face. We shall address it in the next section.

Assuming risk neutrality, the observed Italian instantaneous forward rate for date $T$ is thus a weighted average of two instantaneous forward rates: the German one and that of Italy if it is out of the EMU. The weights are the probability that at date $T$ Italy belongs to the EMU, or is out of the EMU, respectively. In symbols:

$$
f(T)_{t}=\pi(T)_{t} f^{*}(T)_{t}+\left(1-\pi(T)_{t}\right) f^{\text {out }}(T)_{t}
$$

where $f(T)_{t}$ is the Italian instantaneous forward rate for date $T$, observed at date $t, f^{*}(T)_{t}$ the German instantaneous forward rate for date $T$, observed at date $t$, $f^{\text {out }}(T)_{t}$ the Italian instantaneous forward rate for date $T$, if at that future date Italy does not belong to EMU, estimated on the basis of information available at date $t$, and $\pi(T)_{t}$ is the probability that at time $T$ Italy belongs to the EMU, evaluated on the basis of information available at time $t^{6}$

\footnotetext{
${ }^{5}$ See Svensson (1994).

${ }^{6}$ Throughout the paper, $\pi(T)_{t}$ refers to the event 'Italy is inside EMU at date $T$ '. The probability of entering exactly at date $T$, for $T>$ January 1,1999 , is the time derivative of $\pi(T)_{t}$ with respect to $T$. Thus, $1-\pi(T)_{t}$ is similar to a survival function, while the probability of entering is similar to a hazard rate. Similar but not identical, because $\pi(T)_{t}$ does not necessarily converge to $l$ asymptotically as $T$ goes to $\infty$, neither in theory nor in the data.
} 
Let $d(T)_{t} \equiv f(T)_{t}-f^{*}(T)_{t}$ and $d^{\text {out }}(T)_{t} \equiv f^{\text {out }}(T)_{t}-f^{*}(T)_{t}$. Then, (1) can be rewritten as

$$
d(T)_{t}=\left(1-\pi(T)_{t}\right) d^{\text {out }}(T)_{t}
$$

which implies that the estimated probability that at date $T$ Italy belongs to the EMU is

$$
\hat{\pi}(T)_{t}=1-\frac{d(T)_{t}}{d^{\text {out }}(T)_{t}} .
$$

Thus, given an estimate of $f^{\text {out }}(T)_{t}$ for any future date $T$, and given the observed Italian and German instantaneous forward rates, it is easy to compute the probability assigned by financial markets to the event that Italy belongs to the EMU at that date. Fig. 1 illustrates the idea graphically.

The $f *(96: 1)$ curve is the German instantaneous forward rate observed on March 31, 1996. The $f(96: 1)$ curve is the Italian instantaneous forward rate observed on March 31,1996 . The $f^{\text {out }}(96: 1)$ curve is our estimate of $f^{\text {out }}(T)_{t}$ namely of the Italian instantaneous forward rate if out of the EMU. Any point on the dotted line after December 31,1998, is a weighted average of the two solid curves, with weights that vary over time. The weight on the German curve is the probability that Italy will be inside the EMU at that date. As expected, this probability increases over time. The next section explains how these three curves can be estimated.

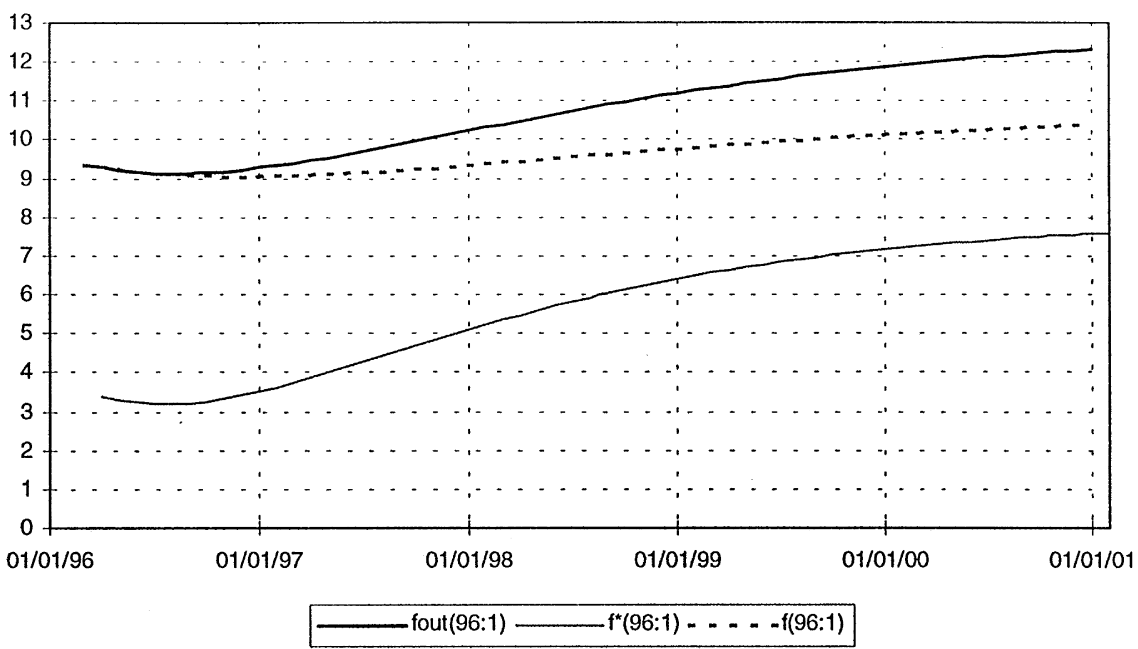

Fig. 1. Forward rates and convergence to EMU, as of March 31st 1996. 


\section{Forward curves and probabilities of entry into the EMU}

In the previous section we have shown that in order to identify the probabilities we must estimate three instantaneous forward curves: the Italian instantaneous forward curve, the German instantaneous forward curve, and the Italian forward curved if out of the EMU. We estimate the first two curves using the Nelson-Siegel interpolant discussed in Svensson (1994) - details of this estimation are provided in the appendix. This section discusses our estimate of the $f^{\text {out }}$ curve and computes the probabilities of entry into the EMU.

The basic idea behind our estimate of $f^{\text {out }}$ is that if Italy had been left out of the EMU, then it would have reverted to the same monetary regime that existed at earlier dates, before the convergence process began, namely an ERM with some room for exchange rate fluctuations. ${ }^{7}$ In such a regime, exchange rate stability is an important goal for monetary policy, yet there is some scope for pursuing domestic macroeconomic goals. The Italian short-term rate is thus determined both by the level of the German short-term rate and by macroeconomic fundamentals in Italy relative to Germany. Our estimate of $f^{\text {out }}$ thus entails two steps. First, we estimate a policy reaction function over some earlier period, that maps macroeconomic variables into Italian short-term interest rates. We assume that the estimated coefficients of this policy reaction function would apply if Italy is out of the EMU. Second, we take 'Consensus' forecasts of the future values of macroeconomic variables entering the policy rule, and we compute the implied Italian short-term interest rate on the basis of the estimated policy rule. We discuss this assumption later in this section.

The reaction function for Italian monetary policy is estimated on quarterly data over the sample 1987:1-1996:2. The starting date was selected because it refers to a period in which Italian inflation and budget deficits were still high relative to the rest of Europe, though monetary policy was giving considerable weight to the goal of exchange rate stability. The sample ends just before the announcement of the very tight budget for 1997, and before the start of the rapid convergence of Italian interest rates towards those of Germany. The obvious dependent variable one should use in estimating our policy rule should be the overnight rate, which is the observable equivalent of the instantaneous forward rate. As shown in Fig. 2, however, the Italian overnight rate is extremely volatile - a result of the reserve requirement regime prior to the reform of October $1990 .{ }^{8}$ In fact, only from the end of 1990 onwards, the overnight rate moves closely to other short-term rates, such as the 3-month euro rate. We have thus specified our reaction function on 3-month euro rates.

\footnotetext{
${ }^{7} \mathrm{We}$ therefore rule out the return to a high-inflation regime.

${ }^{8} \mathrm{Up}$ to October 1990 Italian banks were forced to meet the mandatory reserve requirement daily, rather than on average over the maintenance period.
} 


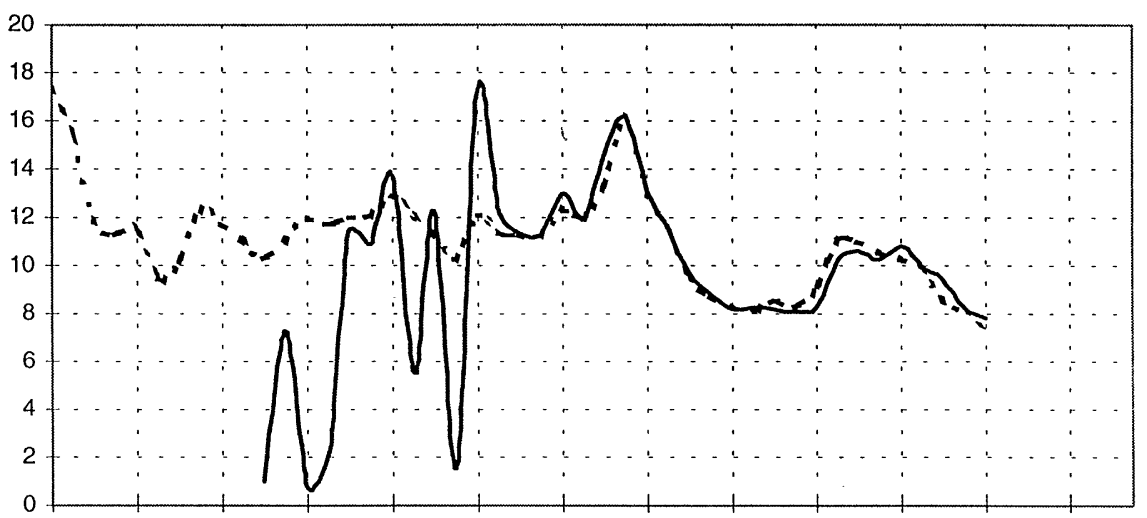

1986-1 1987-1 1988-1 1989-1 1990-1 1991-1 1992-1 1993-1 1994-1 1995-1 1996-1 1997-1

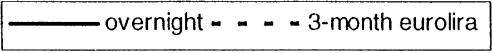

Fig. 2. The Italian overnight rate and the 3-month eurolira rate (quarterly data, end of period).

In estimating such a policy reaction function and assuming that it applies if Italy is out of the EMU, several giant leaps of faith are needed. On the one hand, the period 1987-1996 is rich in events that could have caused instability in the estimated policy rule: in 1990 German reunification took place; in 1992 Italy was forced out of the ERM; the Maastricht Treaty was signed in 1991, and in the last few years of our sample monetary policy goals could have changed reflecting the Maastricht convergence criteria; since 1995 the Bank of Italy has announced explicit inflation targets, presumably giving them more weight than in the past. We discuss below the stability of our estimates, and how we cope with these potential problems. On the other hand, January 1, 1999 marks a fundamental change in the European landscape, and it is likely that this would be reflected in Italian monetary policy if out of the EMU. The past, in this case, is inevitably a bad predictor of future events. There is no way around these problems. We note, however, that our methodology delivers estimates of $f^{\text {out }}$ forward rates very similar to those used by standard EMU Calculators: thus the difference in estimated probabilities between our paper and these Calculators is not due to this admittedly controversial aspect of our procedure.

The estimated rule is reported in Table $2 .^{9}$ The Italian short-term interest rate depends on its own lag, on the current and lagged levels of the German short-term rate, and on three weakly exogenous variables: the inflation gap

\footnotetext{
${ }^{9}$ Our specification of the equation for short-term interest rates is a rule very much in the spirit of Taylor (1993) and Clarida et al. (1997), although adapted to an open economy.
} 
Table 2

A rule for short-term Italian interest rates

Modelling $i_{t}$ by OLS. The sample is: 1987 (1) to 1996 (2)

\begin{tabular}{lccrc}
\hline Variable & Coefficient & Std. error & $t$-value & $t$-prob \\
\hline Constant & 0.025 & 0.012 & 2.03 & 0.0510 \\
$i_{t-1}$ & 0.600 & 0.126 & 4.72 & 0.0001 \\
$p_{t}-p_{t}^{*}$ & 0.314 & 0.140 & 2.23 & 0.0337 \\
$y_{t}-y_{t}^{*}$ & 0.264 & 0.107 & 2.45 & 0.0203 \\
GEMU dummy & -0.184 & 0.091 & -2.01 & 0.0532 \\
$e_{t}$ & -0.079 & 0.023 & -3.42 & 0.0019 \\
$e_{t-1}$ & 0.047 & 0.022 & 2.10 & 0.0438 \\
$i_{t}^{*}$ & 0.906 & 0.281 & 3.21 & 0.0032 \\
$i_{t-1}^{*}$ & -0.487 & 0.330 & -1.48 & 0.1503 \\
\hline
\end{tabular}

$R_{2}=0.77975, F(8,29)=12.834[0.0000]$, s.e. $=0.007667534, \mathrm{DW}=1.74$.

Diagnostic tests:

AR $1-3 F(3,26)=0.318[0.81]$, ARCH $3 F(3,23)=0.14[0.93]$, Normality $\chi^{2}(2)=10.613[0.0050]$, $* * \Xi^{2} F(16,12)=1.425[0.27]$, RESET $F(1,28)=0.56[0.46]$.

Solved static long-run equation:

$i^{i t}=\underset{(0.029)}{0.063}+\underset{(0.30)}{1.04} i^{*}-\underset{(0.045)}{0.08} e+\underset{(0.30)}{0.78}\left(p-p^{*}\right)+\underset{(0.28)}{0.66}\left(y-y^{*}\right)$.

Modelling $i_{t}$ by OLS. The sample is: 1987 (1) to 1996 (2)

\begin{tabular}{lcccc}
\hline Variable & Coefficient & Std. error & $t$-value & $t$-prob \\
\hline Constant & 0.031 & 0.009 & 3.276 & 0.0028 \\
$i_{t-1}$ & 0.576 & 0.095 & 6.065 & 0.0000 \\
$p_{t}-p_{t}^{*}$ & 0.228 & 0.106 & 2.139 & 0.0413 \\
$y_{t}-y_{t}^{*}$ & 0.234 & 0.081 & 2.900 & 0.0072 \\
GEMU dummy & -0.190 & 0.068 & -2.785 & 0.0095 \\
$e_{t}$ & -0.066 & 0.017 & -3.752 & 0.0008 \\
$e_{t-1}$ & 0.045 & 0.016 & 2.713 & 0.0113 \\
$i_{t}^{*}$ & 1.006 & 0.211 & 4.753 & 0.0001 \\
$i_{t-1}^{*}$ & -0.717 & 0.251 & -2.854 & 0.0080 \\
EMS dummy & 0.031 & 0.006 & 4.879 & 0.0000 \\
\hline
\end{tabular}

$R^{2}=0.880953, F(9,28)=23.022|0.0000|$, s.e $=0.005736896, \mathrm{DW}=1.86$.

Diagnostic tests:

AR $1-3 F(3,25)=1.13[0.3556]$, ARCH $3 F(3,22)=0.25[0.8614]$, Normality $\chi^{2}(2)=0.634888$ [0.7280].

$\Xi^{2} F(17,10)=0.29833[0.9862]$, RESET $F(1,27)=0.175522[0.6786]$.

Solved static long-run equation:

$i=\underset{(0.021)}{0.073}+\underset{(0.22)}{0.68} i^{*}-\underset{(0.029)}{0.048} e+\underset{(0.21)}{0.53}\left(p-p^{*}\right)+\underset{(0.19)}{0.55}\left(y-y^{*}\right)$.

Variables are defined as follows:

$i_{t}^{*}$ : interest rate on three-month EuroDM.

$i_{t}$ : interest rate on three-month Eurolira.

$p_{t}-p_{t}^{*}$ : difference between annual inflation in Italy and Germany.

$y_{t}-y_{t}^{*}$ : difference between annual industrial production growth in Italy and Germany.

$e_{t}: \log$ of the dollar/Deutschemark exchange rate. 
between Italy and Germany, defined as the difference between the headline annual CPI inflation (log of price in quarter $t$ minus $\log$ of price in quarter $t-4$ ) between the two countries; the output gap between Italy and Germany, defined as the difference in annual GDP growth in the two countries; and the current and lagged levels of the log of the dollar-Deutschemark exchange rate. Because of the potential effect of German reunification on our estimated coefficients, we have interacted both the inflation gap and the output gap with a reunification dummy, which takes a value of $l$ throughout 1991, and of zero anywhere else. The reunification effect is significant when interacted with the output gap, but not when interacted with the inflation gap: in the final specification we have thus kept only the product of the output gap and the reunification dummy (GEMU dummy).

The intuition behind our specification is that of a small open-economy Taylor-rule where the central bank has an objective function which includes, along with the usual macroeconomic variables such as inflation and growth, exchange rate stability. The objective of exchange rate stability is implemented by defining the target values of the macroeconomic variables as those assumed by these variables in the reference country - Germany. The lira-Deutschemark rate cannot be assumed to be weakly exogenous: it was thus replaced, in the estimation, with the dollar-Deutschemark rate, which we interpret as a weakly exogenous instrument correlated with the lira-Deutschemark rate (see Giavazzi and Giovannini, 1989). Exogeneity of the macroeconomic variables is guaranteed if monetary policy takes some time - at least one-quarter - to affect such variables - by now a standard assumption in the literature on the monetary transmission mechanism which uses structural VAR models (Bernanke and Mihov, 1998; Christiano and Eichenbaum, 1992; Leeper et al., 1996). ${ }^{10}$

We also experimented with alternative specifications. We included, as righthand-side variables, the Italian GDP gap - deviation of actual GDP from an Hodrick-Prescott trend - and a commodity price index. The GDP gap would be justified if we allow for the possibility that even a small open economy can target

GEMU dummy: dummy for German reunification interacted with the difference in growth between Italy and Germany (taking a value of 1 in 1991 and zero everywhere else),

EMS dummy: dummy for EMS crisis (taking a value of 1 in 1992:4 and zero everywhere else).

$\mathrm{AR}$ is an LM test of the null of absence of autocorrelation of residuals, ARCH and $\Xi^{2}$ are tests of the null of absence of heteroscedasticity, RESET is a test of misspecification due to incorrect functional form, and Normality is a test of normality of residuals. For a complete description of all statistics see Hendry (1995). The solved static long-run equation for a general dynamic model of the type $A(L) y_{t}=B(L) x_{t}+u_{t}$ is obtained by setting $L$, the lag operator, to one.

\footnotetext{
${ }^{10}$ Note that our single-equation rule is consistent with the rules derived within VAR models: under our identifying assumptions, the parameters estimated in a structural VAR would coincide with the ones delivered in our single-equation framework.
} 
growth independently of the reference country; the commodity price index has an established tradition as a leading indicator of inflation, and is a relevant argument in reaction functions of central banks. ${ }^{11}$ Neither the deviation of GDP from its trend, nor commodity prices turn out to be significant when added to our basic specification. On the basis of these results we concluded that there is no statistical evidence that the Italian central bank targets the deviation of home GDP from its trend (as identified by the HP filter): we thus omitted this variable from the estimated equation. We also omitted commodity prices: as we include the German policy rate, and considering that this rate reacts significantly to commodity prices (Bernanke and Mihov, 1997; Clarida and Gertler, 1996), we concluded that commodity prices would not play an independent role in our specification. Finally, we also included a measure of fiscal policy: in Italy the exchange rate and more generally exchange rate expectations react to the perceived sustainability of fiscal policy (see Sargent and Wallace (1981) for a theoretical argument along these lines). Since exchange rate stability was certainly a policy goal during the estimation period, it is likely that the Bank of Italy would have responded to fiscal policy news to the extent that these were associated with expected depreciation. As a measure of fiscal policy, we used seasonally adjusted quarterly budget deficits as a percent of GDP. They never were statistically significant, and we thus omitted this variable from the final specification. The quarterly budget deficit is probably not a good indicator of the perceived sustainability of the government budget, but we could find no better observable and exogenous measure of budgetary sustainability. Table 2 reports our results. The coefficient on the lagged dependent variable is 0.6 and that on short-term German interest rates is not significantly different from one. The coefficients on the inflation and output gaps are, respectively, 0.31 and 0.26 in the short run, 0.78 and 0.66 in the long run. These long-run coefficients are significantly different from zero, but not from the values originally chosen by John Taylor. Both the short- and the long-run coefficient on the dollar-Deutschemark exchange rate take a value of -0.08 , implying that a one percentage point appreciation of the dollar against the Deutschemark will be reflected in an eight basis points fall in the Italian short-term rate. The estimated equation passes all the diagnostics with the exception of the normality of residuals. As shown in Fig. 3, which displays the time series of actual and fitted Italian short-term rates, the absence of normality in the residuals may be due to the presence of an outlier in 1992:4. This is likely to be associated to a temporary shock in interest rates related to the unusual behaviour of financial markets

\footnotetext{
${ }^{11}$ The original Taylor rule does not include commodity prices as an explanatory variable in the determination of short-term interest rates. However, the structural VAR approach has shown that the omission of this variable may lead to mis-specification of the reaction function, and to some puzzling impulse responses, i.e. prices declining in response to an expansionary monetary policy shock. See, e.g. Christiano et al. (1996, 1997).
} 


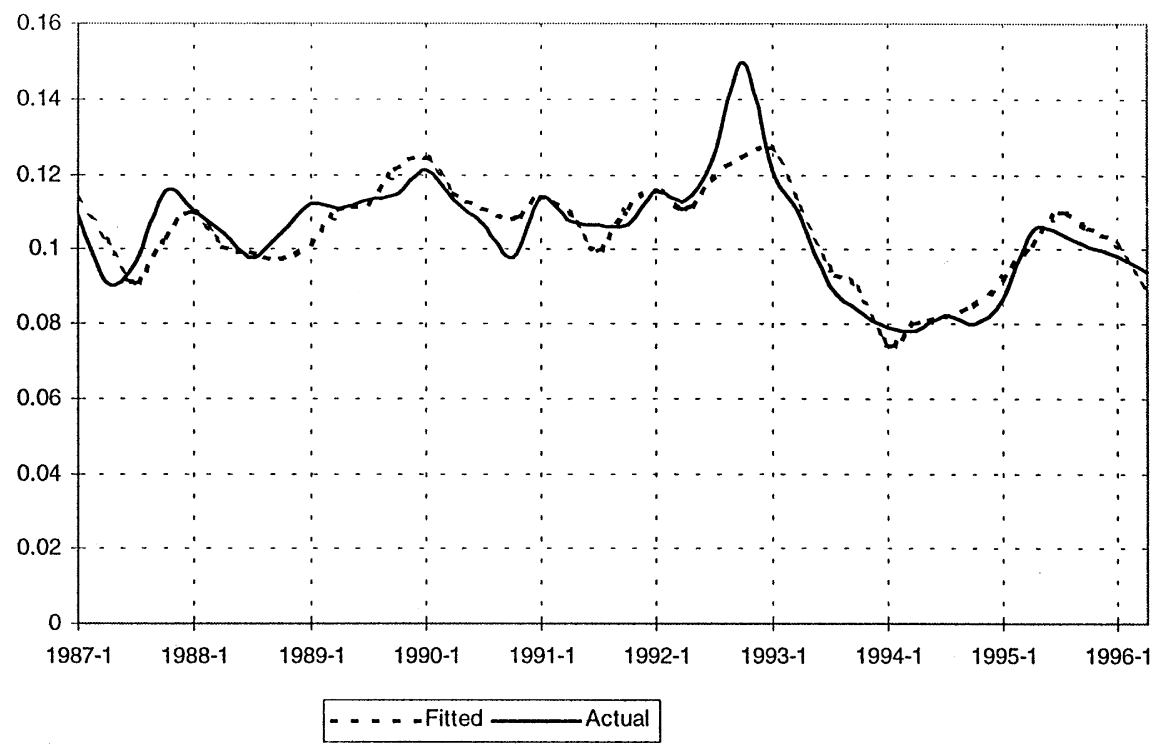

Fig. 3. Actual and fitted Italian 3-month eurolira rates (quarterly data, end of period).

during the EMS crisis, rather than to a structural break in the model. This suspicion is confirmed by the results, also reported in Table 2, obtained including a point dummy for 1992:4. Including the dummy eliminates the nonnormality problem, but does not alter the other coefficients.

To further investigate the effects of temporary shocks to interest-rates we have simulated a shock of 315 basis points - the value of the coefficient on the EMS crisis dummy. The inflation and output gaps, the dollar-Deutschemark rate, and the German three-month rate are all set at their observed values in 1997:1. The model is initialised at the steady state. The simulation, reported in Fig. 4, shows that a shock to short-term interest rates of the dimension observed on the occasion of the Italy's exit from the ERM is re-absorbed within two years.

Having estimated the rule for Italian short-term rates up to 1996:2, we proceed as follows:

- we map this rule into the $f^{\text {out }}$ curve, projecting it forward for two years. We use 'Consensus' forecasts for Italian and German inflation and growth, and for the dollar-Deutschemark exchange rate. This is valid under the assumption that output and inflation move slowly over time so that their values in the near future (up to two years ahead) are not greatly affected by whether or not Italy is a member of the EMU. The path of short-term German rates is instead derived from the estimated German instantaneous forward curve; 


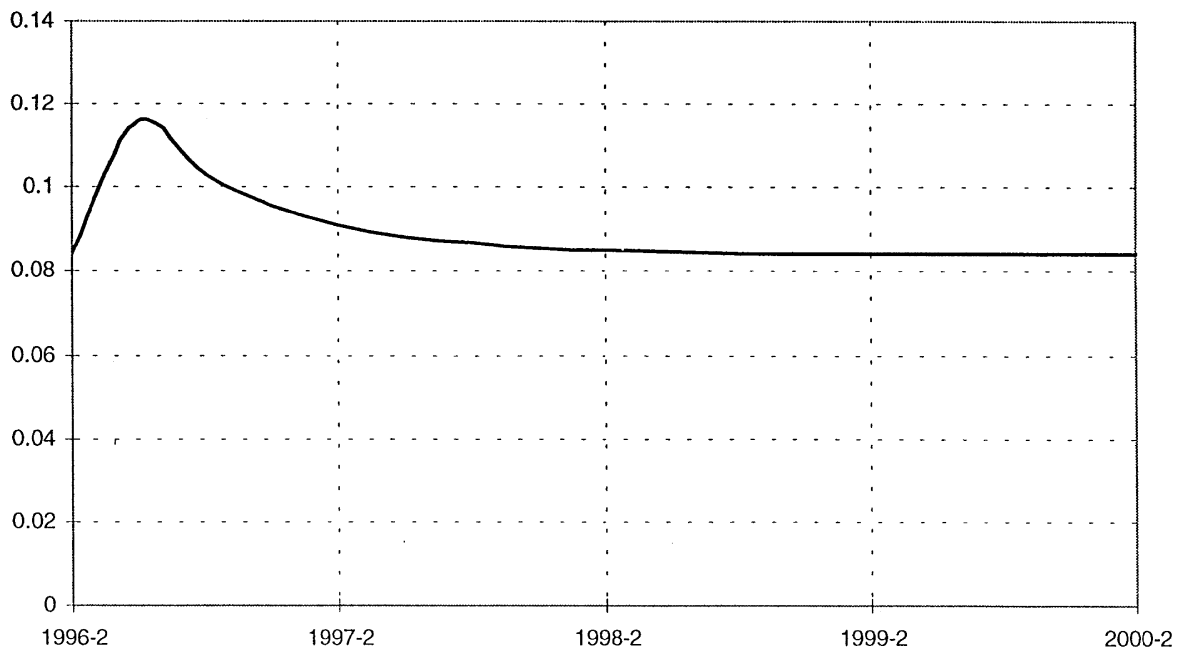

Fig. 4. Simulating the effect of a 315 basis points shock to the risk premium on the 3 -month eurolira rate (steady state $=0.0842$ ).

- we fit a Nelson-Siegel forward function through 10 points: the current overnight rate, the eight rates obtained projecting the rule as described above, and an asymptote which corresponds to the long-run solution of the estimated rule. The 10 points thus obtained are sufficient to identify the four parameters in the Nelson-Siegel forward function.

This procedure allows us to map expected fundamentals into an $f^{\text {out }}$ curve, and thus to associate the level of the $f^{\text {out }}$ Italian interest rate to the evolution of fundamentals. Except for the German forward rate, all these fundamentals are obtained from the Consensus forecasts formulated every quarter from 1995:4 onwards with a forecasting horizon extending over the following four quarters. From then on $f^{\text {out }}$ reflects the asymptotic solution of the Nelson-Siegel forward function.

As was the case with our estimates of the policy reaction function, relying on consensus forecasts to generate values of future macroeconomic variables entails some questionable assumptions. Our last consensus forecast for Italian inflation refers to 1998:1. This last observation is then extrapolated in the indefinite future to construct the asymptotic solution of $f^{\text {out }}$. We are thus assuming that financial markets do not envisage an increase in inflation as a result of Italy being out of the EMU, compared to the consensus forecast referring to 1998:1. This forecast, however, could be kept low by the expectation of ongoing convergence to satisfy the Maastricht criteria, and thus could not be a good measure of expected inflation conditional on Italy being out of the EMU. However, our maintained 
assumption is that, even if not admitted into the EMU from the beginning, Italy would have pursued convergence, aiming for entry at a later date. In Sections 4 and 5 we check the robustness of our result with respect to alternative definitions of $f^{\text {out }}$, and we further discuss our assumptions in light of the empirical findings.

We now have all the information needed to compute the probabilities: we show in Fig. 5a-c the estimated curves for the actual Italian forward rate, the German forward rate, and the Italian $f^{\text {out }}$ forward rate, at several different dates during 1996 and 1997 (the dates correspond to those of the Consensus Forecasts). On the basis of these curves, we compute the probabilities that Italy belongs to the EMU at two future dates: January 4, 1999 and January 1, 2001. These probabilities are reported in Table 3 . Note that $\pi(T)_{t}$ is the unconditional probability of being inside EMU at date $T$. We can also compute the probability of being inside EMU at date $T$, conditional on being out at some earlier date $S<T$. This conditional probability is simply $\left(\pi(T)_{t}-\pi(S)_{t}\right)$ : it measures the probability of entering EMU after date $S$ but not after date $T$. This conditional probability is also displayed in Table 3, with reference to the dates January 1999 and January 2001.

Fig. 6 displays the probability, computed on March 10, 1997, of Italy being inside EMU at any date between January 1999 and April 2002. As expected, the probability increases over time and converges asymptotically to 0.5 . Note that the slope is practically flat after January 2001, indicating that the probability of entry after that date is perceived by financial markets to be very small. In other words, in March 1997 Italy was still perceived as a likely late entrant - the probability of Italy being inside the EMU is always below $50 \%$ - but the delay in Italy's entry was perceived to be short: two-year at most. ${ }^{12}$

Several features of our results are worth commenting. First, the substantial decrease in Italian forward rates from the beginning of our sample to March 1997 is mostly associated with a decrease in the $f^{\text {out }}$ forward rates rather than with an increase in the probability of Italy being inside EMU from the start. From March to October 1997, on the other hand, the $f^{\text {out }}$ rate remains practically constant while $\pi$ jumps up. Such evidence suggests that the $f^{\text {out }}$ variable is independent of the probability of convergence. Second, the probabilities of Italy joining in 1999, computed according to our procedure, are uniformly lower than those derived using the EMU Calculators presented in Table 1: the probabilities

\footnotetext{
${ }^{12}$ If our assumption of risk neutrality were incorrect, our measure of the probability of being inside the EMU could be biased. Bates (1998) assesses the expectational hypothesis of the determination of interest rates and interest rate spreads in Europe. He finds that the data support the hypothesis, particularly in the case of interest rate spreads. He also examines other potential weaknesses of our methodology: the use of interest rates, rather than bond prices, and our assumption that investors make their computation using the lira as the reference currency. He finds them to be of limited empirical relevance.
} 

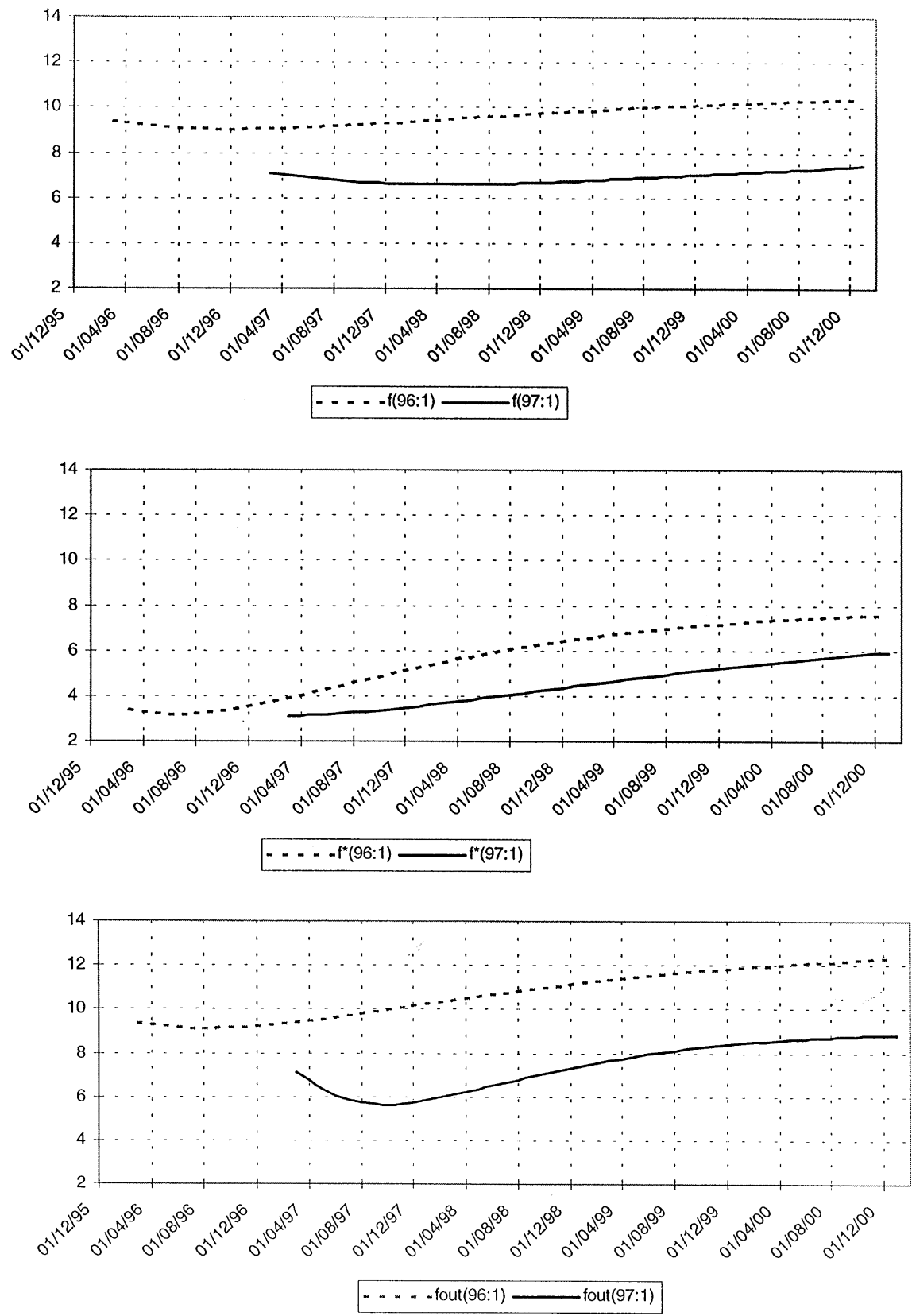

Fig. 5. (a) Italian instantaneous forward rate curves at different dates. (b) German instantaneous forward rate curves at different dates. (c) Italian instantaneous forward rate curves associated, at different dates, to the event Italy does not join the EMU. 


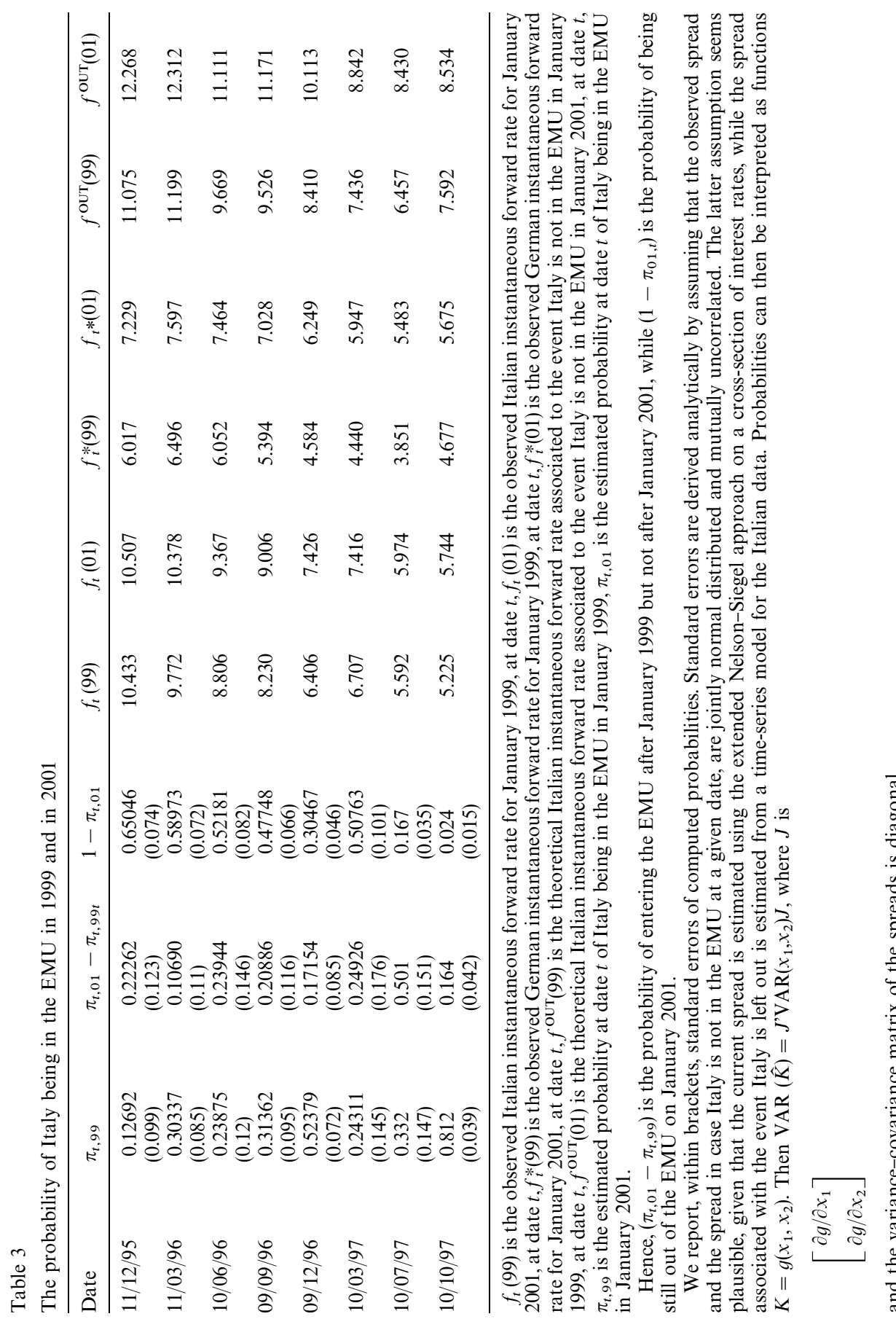




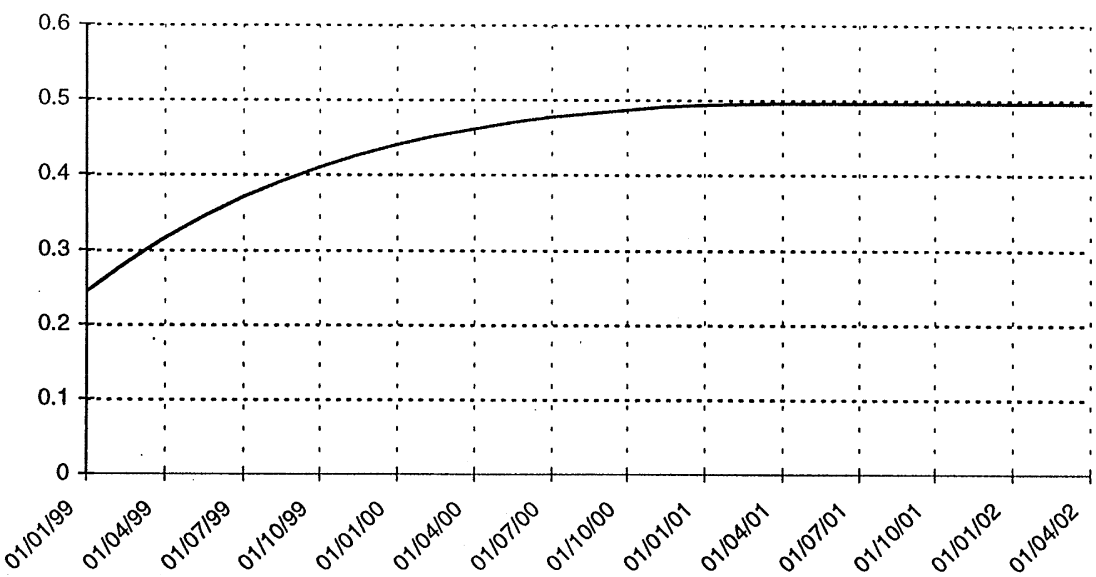

Fig. 6. Probability of Italy being in the EMU at different future dates as measured on March 10, 1997.

computed using either Calculator (shown in Table 1) are constantly outside the $95 \%$ confidence interval centred around our own estimates. Third, the probabilities of Italy being inside the EMU by 2001 are much closer, and in many cases not statistically different, from the probabilities of convergence in 1999 computed by the EMU Calculators. We shall devote the next two sections to a closer investigation of these results.

\section{Convergence of probabilities or convergence of fundamentals?}

Does the observed convergence of Italian and German interest rates, observed from the second-half of 1996 onwards, depend on the convergence of fundamentals (inflation and output gaps), or is it the result of an increase in the probability that Italy joins the EMU? Angeloni and Violi (1998) have recently proposed a methodology that allows to identify the relative contribution of these factors. They show that the reduction in the spread between Italian and German interest rates is more related to the convergence of fundamentals than to a change in the market assessment of the probability of Italy joining the EMU. Here we adapt and extend their methodology to investigate the same issue within our framework.

Differentiating (2), for any future date $T$ we can decompose the time variation of the forward spread between Italy and Germany in its components:

$$
\Delta d(T)_{t}=\left(1-\pi(T)_{t}\right) \Delta d^{\text {out }}(T)_{t}-d^{\text {out }}(T)_{t} \Delta \pi(T)_{t} .
$$


The term $\left(l-\pi(T)_{t}\right) \Delta d^{\text {out }}(T)_{t}$ captures that part of the change in the spread related to domestic fundamentals, since they affect the spread if out of EMU. The term $\left[-d^{\text {out }}(T)_{t} \Delta \pi(T)_{t}\right]$ captures instead the change in markets' perception of the probability of being inside the EMU, and it is thus not directly related to fundamentals.

Consider again the two dates of January 1999 and 2001. The top panel of Table 4 shows that the forward spread between Italy and Germany for January 1999 dropped from 441 basis points at the end of 1995, to 226 basis points at the end of the first-quarter of 1997 and to 55 basis points in October 1997. Similarly, the forward spread for January 2001 decreased form 328 basis points to 147 by March 1997 and to 7 basis points by October 1997. Note, however, that both measures of $d^{\text {out }}$, fall until March 1997 and remain almost flat thereafter. The lower panel of Table 4 shows the decomposition period by period, and over the entire interval. We can identify two different periods: up to March 1997 the overall reduction in the forward spread for January 4, 1999 amounts to 214 basis points. Of these, 149 basis points can be attributed to the direct effect of fundamentals, while 65 to the probability effect. Similarly, out of a total reduction of 181 basis points in the forward spread for January 1, 2001, 109 are accounted for by fundamentals, and only 72 by the probability effect -64 accounted for by a change in the probability of Italy converging in 1999 , and 8 by a change in the probability of Italy converging in 2001. These numbers suggest that the observed convergence between Italian and German interest rates over this period is due to an improvement in Italian fundamentals rather than to an increase in the probability of joining EMU. The situation changes after the summer of 1997: from then on convergence depends almost entirely on the market assessment of probabilities while relative fundamentals remain virtually unchanged.

Concluding, our results suggest the existence of two different regimes in the Italian convergence process. At the beginning the policy turnaround moved fundamentals and thus interest rates, without significantly affecting the probabilities of Italy joining the EMU. Only later, once fundamentals had converged, the market's assessment did change.

\section{Evaluating EMU calculators}

The probability that Italy joins the EMU in 1999, computed according to the technique we have described, is significantly lower than that computed by J.P. Morgan and Credito Italiano, and reported in Table 1. Why is there a difference, and which technique should be used?

The J.P. Morgan and the Credito Italiano 'EMU Calculators' both use the following assumption to compute the probability that Italy belongs to EMU in 1999:

$$
d(99)_{m, t}=\left(1-\pi(99)_{t}\right) d^{\text {out }}(99)_{m, t}
$$




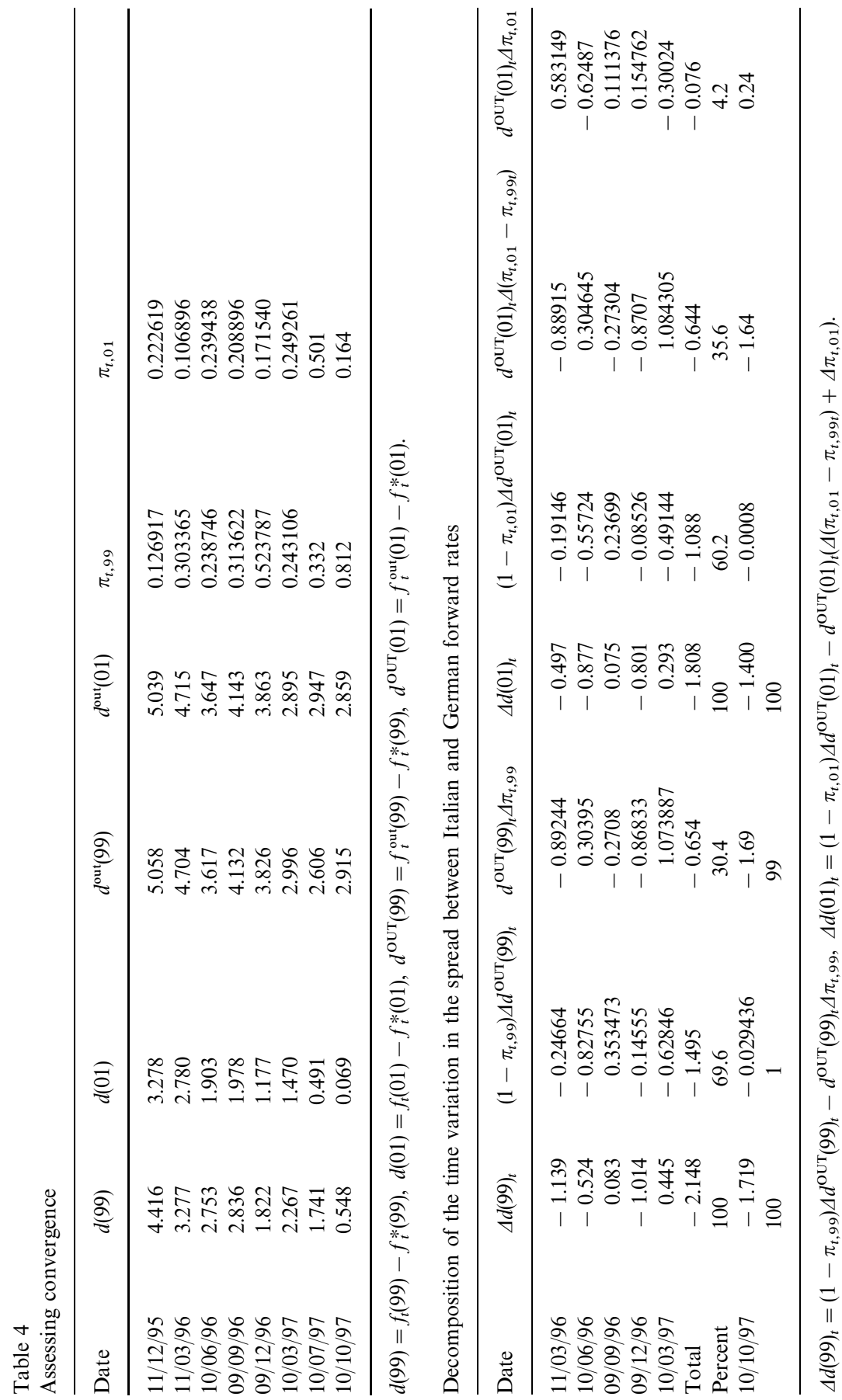


where $d(99)_{m, t}$ is the observed spread between the Italian and German m-year forward rates with settlement in 1999 , and $d^{\text {out }}(99)_{m, t}$ is the theoretical spread between the Italian and German m-year forward rates with settlement in 1999 in the event 'Italy belongs to EMU in 1999'. $m$ is set to three years by Credito Italiano, and to five years by J.P. Morgan.

The two institutions also use different assumptions to pin down the value of $d^{\text {out }}$, the differential in the event Italy does not join the EMU. Credito Italiano sets this spread equal to its average in 1993 - on the presumption that in 1993 the start of the EMU had not yet been incorporated in market prices. J.P. Morgan identifies this spread by claiming that there is an international price for risk which determines the total spread. Therefore 'if EMU were not around, the spread between Italian and German bonds would be highly correlated with international measures of risks such as US-Australia, US-Canada, BradyTreasury, the US long bond-2 year bond spread, as well as the order of performance of non-European currencies and market volatilities' (J.P. Morgan, 1997). J.P. Morgan thus estimates a regression of the observed spread between Italian and German swap rates on average non-European spreads, the level of US market rates, the slope of the US yield curve, and non-European measures of volatility, using daily data over the sample January 1989-December 1991. Using the estimated coefficients of this regression, they map current observations on the regressors into a level of the spread if Italy is left out of the EMU. The value of the spread thus computed can be updated daily, at a much higher frequency than our measure of $d^{\text {out }}$, which is based on macroeconomic fundamentals which can only be updated quarterly.

The choice of a different method for computing $d^{\text {out }}$ is an obvious candidate to explain the divergence between the probabilities we derive, and those derived by the two institutions. We can rule out this possibility. The probabilities computed by J.P. Morgan and Credito Italiano move very close to one another, and the level of $d^{\text {out }}$ based on the measure of the price of risk computed by J.P. Morgan is not very different from that estimated in this paper on the basis of Italian macroeconomic fundamentals. Fig. 7 plots both the J.P. Morgan measure of the 2-year forward differential for January 4, 1999, estimated at different future dates $t, d^{\text {out }}(99)_{2, t}$, as well as the corresponding value of $d^{\text {out }}(99)_{2, t}$ used in this paper. To compute our measure of $d^{\text {out }}(99)_{2, t}$ we have converted the instantaneous forward differential, estimated as described in Section 3, in a 2-year forward differential. The difference between our measure and that of J.P. Morgan never exceeds 30 basis points within the sample.

The reason why the probabilities estimated by the two financial houses differ from our own is another one: they use forward rates on contracts maturing some years after the settlement date. In this paper, instead, we use instantaneous forward rates, that is, in practice, contracts with settlement some time in the future and maturity one day after. Credito Italiano uses forward rates with settlement some time in the future, and maturity 3 years after settlement; 


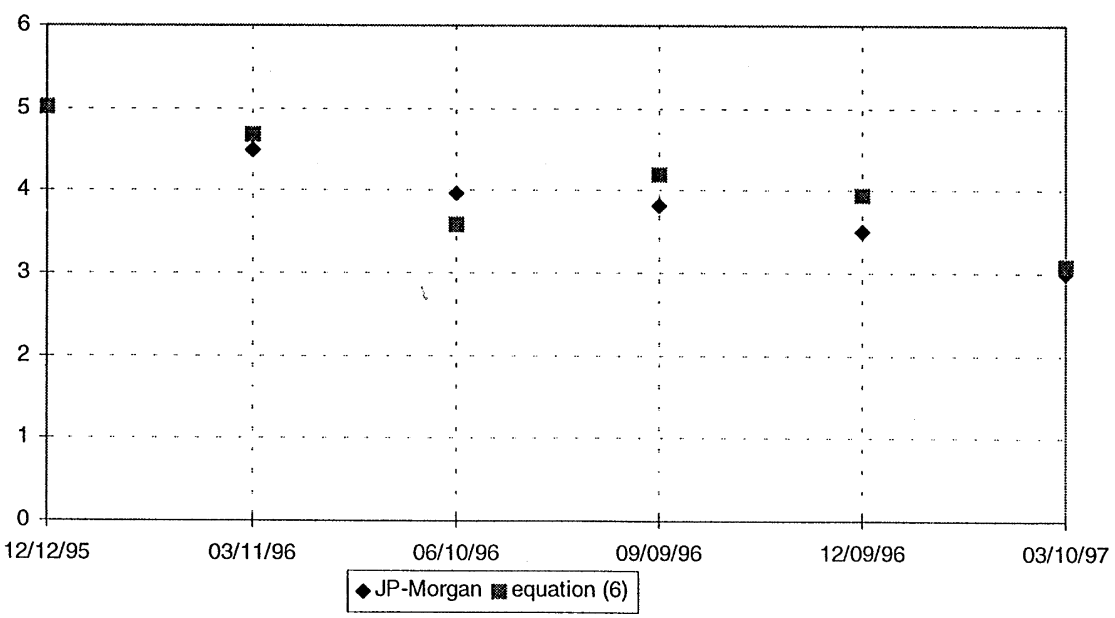

Fig. 7. 2-year forward differentials assuming that Italy does not join the EMU. J.P. Morgan estimates and estimates based on Eq. (A.6).

J.P. Morgan with maturity 5 years after settlement. This implies that the probability computed by these institutions refers to a different event. We estimate the probability that Italy belongs to the EMU at a specific moment in time, say January 4, 1999. These two institutions instead estimate the average of the probabilities that Italy belongs to the EMU at all points in time between date $t$, say January 4, 1999, and date $t+m$. Since the probability of being inside the EMU at a future date $T$ increases with $T$, this measure clearly overestimates the probability of being inside the EMU exactly on January 4, 1999.

To see this point more clearly, recall that the $m$-year forward spread is the average of instantaneous forward spreads over the relevant period. Specifically,

$$
d(T)_{m, t}=\frac{1}{m} \int_{T}^{T+m} d(z)_{t} \mathrm{~d} z
$$

and similarly for $d^{\text {out }}(T)_{m, t}$. These two financial institutions estimate the probability that Italy belongs to EMU at date $T$ as

$$
\pi(\widehat{T})_{t}=l-\frac{d(T)_{m, t}}{d^{\text {out }}(T)_{m, t}} .
$$

By (2) and (6), this estimate reduces to

$$
\pi(\widehat{T})_{t}=\frac{\frac{1}{m} \int_{T}^{T+m} \pi(z)_{t} d^{\text {out }}(z)_{t} \mathrm{~d} z}{\frac{1}{m} \int_{T}^{T+m} d^{\text {out }}(z)_{t} \mathrm{~d} z} .
$$


That is, the estimated probability of being inside the EMU at date $T$ is a weighted average of the probabilities of being inside the EMU between dates $T$ and $T+m$, with $d^{\text {out }}(z)$ as the weights. Credito Italiano assumes that $d^{\text {out }}(z)_{t}$, does not vary with the settlement date, $z: d^{\text {out }}(z)_{t}=d_{t}^{\text {out }}$ for all $z$. In this case (8) reduces to

$$
\pi(\widehat{T})_{t}=\frac{1}{m} \int_{T}^{T+m} \pi(z)_{t} \mathrm{~d} z,
$$

where the right-hand side of $(9)$ is just the average probability of being inside the EMU between date $T$ and date $T+m$. The probability of being inside the EMU increases over time:

$$
\pi(T+m)_{t}>\pi(\hat{T})_{t}>\pi(T)_{t} .
$$

Thus, the probability computed from this EMU calculator based on $m$-year forward rates overestimates the true probability of being inside the EMU at date $T$, the more so the larger is the maturity date $m$. J.P. Morgan, on the other hand, assumes that $d^{\text {out }}(z)_{t}$ increases linearly with the settlement date $z$. In this case the bias in the estimated probability is even greater. The reason is that, by (8), the values of $\pi(z)$ with higher $z$ have larger weights, and, as noted above, $\pi(z)$ increases in $z$. Thus,

$$
\frac{\frac{1}{m} \int_{T}^{T+m} \pi(z)_{t} d^{\text {out }}(z)_{t} \mathrm{~d} z}{\frac{1}{m} \int_{T}^{T+m} d^{\text {out }}(z)_{t} \mathrm{~d} z}=\frac{\frac{1}{m} \int_{T}^{T+m} \pi(z)_{t} z \mathrm{~d} z}{\frac{1}{m} \int_{T}^{T+m} z \mathrm{~d} z}>\frac{1}{m} \int_{T}^{T+m} \pi(z)_{t} \mathrm{~d} z,
$$

where the equality follows from the assumption that $d^{\text {out }}(z)$ increases linearly in $z$. Again, the bias in these estimates is larger the greater is the maturity date $m$. Only if $m$ is zero, as with instantaneous forward rates, and if $d^{\text {out }}(T)_{t}$ is correctly estimated, the estimate of the probability is unbiased.

Consider, as an example, March 10,1997, when our methodology delivers an estimate of 0.24 for the probability of being inside the EMU on January 1, 1999, and 0.48 for the probability of being inside the EMU by January 1, 2001. If we computed the same probabilities using 2-year average forward rates, instead of instantaneous forward rates, without any change in the scenario if out of the

Table 5

Probabilities (as of March 10, 1997) of Italy joining the EMU on Jan 4, 1999

\begin{tabular}{lllll}
\hline $\begin{array}{l}\text { Reuters } \\
\text { EMU } \\
\text { survey }\end{array}$ & $\begin{array}{l}\text { Credito Ital. } \\
\text { EMU calculator }\end{array}$ & $\begin{array}{l}\text { J.P. Morgan } \\
\text { EMU calculator }\end{array}$ & $\begin{array}{l}\text { Our methodology } \\
\text { using instantaneous } \\
\text { forward rates }\end{array}$ & $\begin{array}{l}\text { Our methodology } \\
\text { using average 2-year } \\
\text { forward rates }\end{array}$ \\
\hline 0.12 & 0.46 & 0.57 & 0.24 & 0.41 \\
\hline
\end{tabular}


EMU, the resulting probability of entry in 1999 would be 0.41: an upward bias of $0.17(=0.41-0.24)$. A full comparison of the results provided by alternative 'EMU Calculators' for March 10, 1997 is shown in Table 5. Clearly, the different methodologies yield quite different results.

\section{Conclusions}

We have proposed a new method for extracting from the term structure of interest rates information about the probability that a country joins the EMU at given dates. We have derived two main results.

First, we have estimated the $d^{\text {out }}$ spread between Italian and German instantaneous interest rates, that is the spread that would arise at some date $t$ if, at that date, Italy did not belong to the EMU, by estimating a reaction function for the Bank of Italy along the lines of a 'Taylor rule'. We are thus able to identify two separate components of the total spread between Italian and German interest rates: one is the market assessment of the probability that the country will join the EMU in 1999; the other reflects the fundamentals that would have shaped the spread if the country had been left out of the EMU. For example, the spread between Italian and German forward rates with maturity in 1999 dropped by 215 basis points in the period December 1995 to March 1997. Of these, 150 basis points can be attributed to the convergence of fundamentals, while only 65 to a change in the markets assessment of the probability of Italy joining the EMU in 1999. Between March 1997 and October 1997, the spread dropped by another 172 basis points. This reduction is almost entirely due to an increase in the probability of Italy joining the EMU from the start.

Second, we have shown that the probabilities computed by 'EMU Calculators' were upward biased. This is because they are based on average rather than instantaneous forward rates. The methodology used to compute these 'EMU Calculators' is similar to our own, except for two aspects: (i) we use instantaneous forward rates, while 'EMU Calculators' use forward rates on instruments with much longer maturities; (ii) our estimate of the $d^{\text {out }}$ spread is based on a different technique. Despite the different methodology, our estimate of the $d^{\text {out }}$ spread is very similar in magnitude to that used to construct the 'EMU Calculators'. Yet, the estimated probabilities of joining the EMU are strikingly different. Hence, this difference is almost entirely due to the use of instantaneous rather than average forward rates. The use of instantaneous forward rates is crucial because it provides an easy way to describe the complementary events 'Italy belongs to EMU' and 'Italy is out of EMU' at a specific point in the future. With $m$-period average (rather than instantaneous) forward rates, one estimates the average of the probabilities of being inside the EMU at all points in time during an interval of length $m$. Since the probability of being inside the EMU at a future date $T$ increases in $T$, this average of probabilities is greater than the 
probability of being inside the EMU exactly at the start of the interval considered.

\section{Acknowledgements}

We thank Ignazio Angeloni, Jesper Lund, Ben McCallum, Alessandro Missale, Mario Seghelini and two referees for helpful comments. A version of this paper was presented at the CIDE-Banca dItalia conference on 'Quantitative Methods for Economic Policy'.

\section{Appendix A. Spot rates, the expectational model and forward rates}

To illustrate our derivation of spot and forward rate functions, let us start by considering a zero coupon bond issued at time $t$, with a face value of $l$, maturity of $m$ years, and price $P Z C_{m t}$. The simple yield $Y_{m t}$ is related to the price as follows:

$$
P Z C_{m t}=\frac{1}{\left(1+Y_{m t}\right)^{m}},
$$

Define the spot rate $r_{m t}$ as $\log \left(1+Y_{m t}\right)$, which is the continuously compounded yield, and define the discount function $D_{m t}$ as the price at time $t$ of a zero coupon that pays one unit at time $t+m$. We then have

$$
P Z C_{m t}=\exp \left(-m r_{m t}\right)=D_{m t} .
$$

Consider now a coupon bond that pays a coupon rate of $c$ percent annually, and pays a face value of $l$ at maturity. The price of the bond at trade date is given by the following formula:

$$
P_{m t}=\sum_{k=1}^{m} c D_{k t}+D_{m t} .
$$

Given the prices of coupon bonds, spot rates on zero-coupon equivalents can be derived by fitting a discount function based on the following specification for the spot rates:

$$
\begin{aligned}
r_{k t}= & \beta_{0}+\beta_{1} \frac{1-\exp \left(-k / \tau_{1}\right)}{k / \tau_{1}}+\beta_{2}\left(\frac{1-\exp \left(-k / \tau_{1}\right)}{k / \tau_{1}}-\exp \left(-\frac{k}{\tau_{1}}\right)\right) \\
& +\beta_{3}\left(\frac{1-\exp \left(-k / \tau_{2}\right)}{k / \tau_{2}}-\exp \left(-\frac{k}{\tau_{2}}\right)\right) .
\end{aligned}
$$


The above specification has been originally introduced by Svensson (1994) as an extension of the parametrization proposed by Nelson and Siegel (1987). Note that our estimated spot rate differs from the yield to maturity often quoted for coupon bonds. In fact the quoted yield to maturity, $y_{m t}$, is defined by the following relation:

$$
P_{m t}=\sum_{k=1}^{m} c \exp \left(-k y_{m t}\right)+\exp \left(-m y_{m t}\right) .
$$

Yield to maturities are averages of spot rates up to the date of maturity. While in general spot rates defined by (A.3) vary with the maturity, the yield to maturity defined by (A.5) is constant. Henceforth, the term structure of interest rates estimated on yields to maturity is only valid when the term structure of spot rates is flat. Moreover, the yield to maturity for a bond with a given maturity depends on the coupon rate, the so-called 'coupon effect'. Spot rates instead are free from such an effect.

Implied forward rates can be computed from spot rates. A forward rate at time $t$ with trade date $t+t$, and settlement date $t+T$ can be computed as the return on an investment strategy based on buying zero-coupon bonds at time $t$ maturing at time $t+T$, and selling at time $t$ zero-coupon bonds maturing at time $t+t^{\prime}$. The forward rate is related to the spot rate according to the following formula:

$$
f_{T+t . t^{\prime}+t . t}=\frac{T r_{T, t}-t^{\prime} r_{t^{\prime}, t}}{T-t^{\prime}} .
$$

The forward rate for a 1-year investment with settlement in 2 years, and maturity in 3 years is thus equal to three times the 3 -year spot rate, minus twice the 2-year spot rate.

The instantaneous forward rate is the rate on a forward contract with an infinitesimal investment after the settlement date:

$$
f_{m t}=\lim _{T \rightarrow m} f_{T+t, m+t, t} .
$$

In practice, we identify the instantaneous forward rate with an overnight forward rate, i.e. a forward rate with maturity one day after the settlement. The relationship between the instantaneous forward rate and the spot rate is then

$$
r_{m t}=\frac{\int_{\tau=t}^{t+m} f_{\tau t} \mathrm{~d} \tau}{m}
$$

or, equivalently,

$$
f_{m t}=r_{m t}+m \frac{\partial r_{m, t}}{\partial m} .
$$


Given specification (4) for the spot rate, the resulting forward function is

$$
\begin{aligned}
f_{k t}= & \beta_{0}+\beta_{1} \exp \left(-\frac{k}{\tau_{1}}\right)+\beta_{2} \frac{k}{\tau_{1}} \exp \left(-\frac{k}{\tau_{1}}\right) \\
& +\beta_{3} \frac{k}{\tau_{2}} \exp \left(-\frac{k}{\tau_{2}}\right) .
\end{aligned}
$$

Therefore, as $k$ goes to zero, the spot and the forward rates coincide at $\beta_{0}+\beta_{1}$, and as $k$ goes to infinity the spot and the forward rate coincide at $\beta_{0}$. The forward rate function features a constant, an exponential term decreasing when $\beta_{1}$ is positive, and two 'hump shape' terms.

We estimate a term structure of spot rates based on the observation of the overnight rate, the euro 1-, 3-, 6- and 12-month rates. We then consider the 2-, 3-, 5-, 7- and 10-year fixed interest rates on swaps. We use euro-rates as spot rates, because these are zero-coupon bonds. We then consider swap rates as the long-term rates to be associated to euro rates. Fixed interest rates swaps facilitate international comparisons because they are not affected by different taxation regimes, and by default risk. By fitting the discount function to the data, and minimizing error in the yield space, we then estimate spot and forward rates.

\section{References}

Angeloni, I., Violi, R., 1998. Long-term interest rate convergence in Europe and the probability of EMU. Temi di discussione, No. 322. Banca d'Italia, Rome.

Bates, D.S., 1998. Financial markets' assessment of EMU. Paper presented at the Carnegie-Rochester Conference, November.

Bernanke, B.S., Mihov, I., 1998. Measuring monetary policy. Quarterly Journal of Economics CXIII, 869-903.

Bernanke, B.S., Mihov, I., 1997. What does the Bundesbank target? European Economic Review 41 (6), 1025-1053.

Butler, C., Cooper, N., 1997. Implicit exchange rate correlations and market perception of the European Monetary Union. Bank of England Quarterly Bulletin, 413-423.

Clarida, R., Gertler, M., 1996. How the Bundesbank conducts monetary policy. Working paper no. 5581, NBER, Cambridge, MA.

Christiano, L.J., Eichenbaum, M., 1992. Liquidity effects and the monetary transmission mechanism. American Economic Review 82 (2), 346-353.

Christiano, L.J., Eichenbaum, M., Evans, C.L., 1996. The effects of monetary policy shocks: Evidence from the flow of funds. Review of Economics and Statistics 78, 16-34.

Christiano, L.J., Eichenbaum, M., Evans, C.L., 1997. Sticky price and limited participation models of money: A comparison. European Economic Review 41 (6), 1201-1249.

De Grauwe, P., 1996. Forward rates as predictors of EMU. Discussion paper no.1556, CEPR, London, UK.

Flood, R.P., Garber, P.M., 1983. A model of stochastic process switching. Econometrica 51 (3), 537-551.

Flood, R.P., Marion, N., 1983. Exchange-rate regimes in transition: Italy 1974. Journal of International Money and Finance 2, 279-294. 
Giavazzi, F., Giovannini, A., 1989. Limiting Exchange Rate Flexibility: The European Monetary System. MIT Press, Cambridge, MA.

Hendry, D.F., 1995. Dynamic Econometrics. Oxford University Press, Oxford.

J.P. Morgan, 1997. EMU Calculator Handbook. Foreign Exchange Research: Technical series,

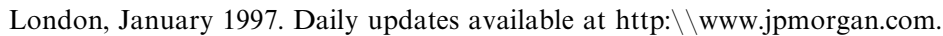

Leeper, E.M., Sims, C.A., Zha, T., 1996. What does monetary policy do? available at ftp://ftp.econ.yale.edu/pub/sims/mpolicy.

Lund, J., 1998. A model for studying the effect of EMU on European yield curves. Working paper No. D97-12, Aarhus School of Business.

Nelson, C.R., Siegel, A.F., 1987. Parsimonious modelling of yield curves. Journal of Business 60, 473-489.

Söderlind, P., Svensson, L.E.O., 1997. New techniques to extract market expectations from financial instruments. Discussion paper no. 1556, CEPR, London, UK.

Svensson, L.E.O., 1994. Estimating and interpreting forward interest rates: Sweden 1992-94. Discussion paper no. 1051, CEPR, London, UK.

Taylor, J.B., 1993. Discretion versus policy rules in practice. Carnegie Rochester Conference Series on Public Policy 39, 195-214.

Weidman, J., 1996. The likelihood of European monetary union. Banca Nazionale del Lavoro Quarterly Review 199, 405-413. 